\title{
GIPOGO ET PUMBU A MFUMU: MASQUES DU POUVOIR CHEFFAL CHEZ LES PENDE DU KASAÏ
}

\author{
Constantijn Petridis*
}

\begin{abstract}
PETRIDIS, C. Gipogo et Pumbu a mfumu: Masques du pouvoir cheffal chez les Pende du Kasaĩ. Rev. do Museu de Arqueologia e Etnologia, S. Paulo, 2:75-89, 1992.
\end{abstract}

RESUMO: O objetivo deste artigo é indicar a dimensão politica de dois tipos de máscaras de madeira dos Pende do Kasaï. São destacados os aspectos formal e funcional. Os limites da pesquisa foram determinados pela literatura disponivel sobre o tema e pela análise morfológica de um vasto número de máscaras. Embora as casas do chefe sejam as realizações artísticas mais impressionantes com relação ao poder chefal Pende, um comportamento dominante, cheio de força, é expresso de uma forma dinâmica nas mascaradas Pende. Entre os Kasaï ou Pende orientais os tipos de máscaras Gipogo e Pumbu a mfumu glorificam e celebram a liderança política para salvaguardar as relaçōes de poder existentes. Como mediadores entre reinos opostos e como forças reguladoras, os mascarados aparecem como extensões da autoridade do chefe. tico.

UNITERMOS: Zaïre. Pende do Kasaï - máscaras - poder polí-

Il est généralement reconnu que l'art africain est étroitement lié tant à la vie religieuse qu'à la vie politique. En fait, dans le context africain, la distinction entre le sacré et le profane s'avère souvent difficile. Dans de nombreux cas, les hommes de pouvoir ne peuvent exercer leurs fonctions que grâce à leur relation particulière avec le surnaturel.

Dans 'African art and leadership', Frase\& Cole (1972) traitent le lien intime entre certaines formes d'art et certains types de pouvoir politique. Les auteurs soulignent l'importance d'une approche comparative et l'utilité des notions de structure, fonction et histoire (1972: 299-300). Ils concluent que l'invariabilité de l'art à travers le temps et l'espace permet une parenté proche entre la personne du chef et l'art. Tandis que le chef constitue

(*) Université de Gand, Belgique. Aspirant du Fonds National Belge pour la Recherche Scientifique. l'aspect dynamique de l'image du pouvcir, l'art en est l'élément statique (1972: 325-326).

La dimension politique de l'art diffère fortement selon la situation spécifique. Fraser \& Cole (1972:296) utilisent deux critères de classification: $1^{\circ}$ le degré de spécialisation; et $2^{2}$ la mesure dans laquelle les rôles, les structures et les systèmes sont autonomes ou au contraire hiérarchisés. 11 est évident que ce sont surtout les systèmes centralisés que se servent d'un granci nombre de symboles pour glorifier et renforc:r le pouvoir établi. Les types d'objets que l'on retrouve le plus fréquemment sont des s‘atues J'ancêtres et des signes de prestige, tels des bátons de pouvoir, des herminettes, des chasse-mouches, des armes, des bijoux et des vêtements. Souvent, les dirigeants politiques soït directement impliqués dans la création artistıque et parfois ils sont même à l'origine de certaines formes ou certains traits iconographiques. 
McLeod (1976: 99) fait une distinction entre deux formes d'art, qu'il décrit comme "statement images", d'une part, et "process images", d'autre part. Il est difficile de donner des traductions adéquates de ces termes. Dans le premier cas, il s'agit d'images qui existent sous une forme achevée et qui, par elles-mêmes, transmettent leur signification. Ces types d'images sont surtout utilisés dans les systèmes politiques centralisés. La deuxième forme a par contre trait à des images qui font partie d'une série d'actions et qui sont faites, modifiées ou abandonnées au cours des temps. En fin de compte, "process images are concerned with alteration and readjustment" (1976: 102).

Cole (1989: 104-105), de son côté, démontre l'importance d'une figure masculine armée et agressive comme icône dominante dans les arts visuels africains. L'icône de l'homme armé est surtout connu à travers les arts statiques, mais on la retrouve également dans les arts dits processuels ou "performatifs". En effet, le pouvoir politique et le comportament mâle agressif sont traduits de façon dynamique dans les représentations masquées.

La situation politique des Pende du Zaïre, qui habitent une vaste région entre les fleuves Lutshima et Kasaï, se caractérise par l'absence d'un pouvoir central. Les Pende n'ont jamais formé une unité politique. Par contre, il existe un nombre de chefferies autonomes dont les chefs ne possèdent que peu de pouvoir effectif et jouent surtout un rôle rituel. Néanmoins, il faut faire une distinction entre le chef de chefferie, fumu a mbanji, et le chef de clan, fumu a giputa ou lemba. ${ }^{1}$ Le chef de chefferie est conçu en quelque sorte comme un prolongement du chef de clan. Il a le pouvoir de vie et de mort et autrefois son investiture était accompagnée d'un homicide. Le crâne de la vic-

(1) Bittremieux (1939: 27) distingue trois types de chefs qui forment une sorte de hiérarchie et ont chacun leurs propres privilèges et attributs: fumu wa mbandi, kilembe et kimuanga. Ces différences terminologiques sont probablement dues à une situation locale de la région de Loanda au Kasaï. D'ailleurs, De Sousberghe (1963: 52) remarque que le caractère et le pouvoir du chef diffèrent considérablement de chefferie en chefferie. Par conséquent, il est presque impossible de tenir compte de toutes ces variantes locales. time était placé sur le sommet de la case cheffale.

La tâche principale du chef de chefferie consiste à veiller au maintien de l'odre et de la paix au sein de la société. Il est assisté à cette fin par l'avocat-orateur, ngambi, par le chef de guerre, ngunza, et surtout par le "ministre", pungu ou punga (De Sousberghe, 1963: 53-54).

Le fumu a mbanji est avant tout un médiateur entre la société des vivants, d'une part, et les forces invisibles et les ancêstres, d'autre part. Il est considéré comme source de vie et de fécondité. Par conséquent, son activité sexuelle est soumise à des règles sévères. On lui donne souvent une vieille femme comme épouse, dans certaines chefferies on lui impose le port d'un étui pénien et dans la région de la Loange une hernie ou enflure scrotale est exigée en signe d'acceptation par les ancêtres (De Sousberghe, 1954:217-218; 1963: 55-59, 66-68).

Des cérémonies complexes accompagnent l'investiture. Souvent, les successeurs éventuels s'enfuient après la mort d'un ancien chef afin d'échapper à la succession. Le nouveau chef est d'abord pris en captivité avec son épouse et est humilié et maltraîté en public. Suit alors une longue période de réclusion dans la forêt, pendant laquelle le chef est initié par le pungu aux tâches et secrets cheffaux. Après la mise à mort d'un ennemi ou d'un passant, le chef est investi officiellement dans sa fonction et porté en triomphe à travers le village. Le fumu a mbanji ne peut mourir de mort naturelle. En cas de maladie ou de vieillesse, il est étranglé par le pungu, aidé par quelques anciens esclaves, ajikulu (De Sousberghe, 1963: 59-61).

L'épouse cheffale, mwata mwadi ou mukaji wa manda, joue un rôle prépondérant dans la vie politique. Originaire d'un clan d'anciens esclaves, elle est aussi la soeur clanique du pungu. La mwata mwadi remplit une fonction précise dans le rite d'investiture et elle est responsable des activités féminines, plus précisément du cycle agraire. Elle réside dans la case cheffale et veille sur le trésor, kifumu. Dans certaines chefferies, le chef dort séparément dans une petite hutte, mbonge ou kolo (De Sousberghe, 1963: 62-65).

Les cases cheffales sont les créations artistiques les plus impressionnantes en relation 
PETRIDIS, C. Gipogo et Pumbu a mfumu: masques du pouvoir cheffal chez les Pende du Kasaĩ. Rev. do Museu de Arqueologia e Einologia, S. Paulo, 2:75-89, 1992.

avec le pouvoir politique. ${ }^{2}$ C'est surtout au Kasaï qu'elles forment de riches ensembles sculpturaux. Le toit de la case du fumu a mbanji est décoré d'une sculpture nommée Gangulungu. Il s'agit d'une statue de femme portant un enfant sur le bras et/ou une calice, bengwa, ainsi qu'une hache, kuba, dans les mains.

Cette statue représente "l'épouse du pouvoir", mukaji wa manda, la première femme du chef qui domine les autres femmes. Autrefois, lors du rite d'investiture, elle exécutait une danse en tenant une hache dans la main. Ensuite, elle passait la hache à son époux nouvellement investi et ce dernier décapitait un chien sacrificiel à l'aide d'un seul coup. En même temps, le pungu coupait la tête d'une chèvre. Le sang des animaux ainsi sacrifiés était récolté dans une calice et bu par les témoins présents en guise d'acceptation du nouveau chef. Ces animaux domestiques remplacent les hommes immolés jadis (De Sousberghe, 1954a: 78).

D'aprés Vandenhoute (1960: 16-17), qui se base sur les données de De Sousberghe, la statue Gangulungu servait à éloigner les maladies, à assurer le pouvoir et à promouvoir la fécondité des gens, des animaux et de la terre. Tout comme le chef politique, la case cheffale aurait une influence directe sur la santé et le bien-être de la société.

Le rapport entre la politique et l'art est également manifeste dans la mascarade pende. Ceci vaut aussi bien pour les masques de la région centrale, dite Katundu, d'après la chefferie du même nom, que pour les masques de la région orientale, le Kasaï. Les deux régions ont leurs styles et formes propres. $\mathrm{Ce}$ sont surtout les masques de style Katundu qui

(2) Au Kasaï, ces cases portent le nom gibulu, tandis qu'en région Katundu on les appelle gisendu. Les panneaux sculpturaux qui entourent la porte d'entrée constituent sans doute l'élément décoratif le plus caractéristique. Au Kasaī, où ces sculptures sont nommés kenene, il $s^{\prime}$ agit le plus souvent d'une statue masculine et d'une statue féminine de chaque côté de la porte et d'une imitation de masque sur le linteau. Dans la région de la rive gauche du Kwilu, la pièce faîtière est $d$ 'habitude une statue de la kumbi, la cigogne à ventre blanc (Sphenorynchus abdimil). En région Katundu, il s'agit d'une statue humaine ou d'une statue animalière (De jousberghe, 1959: 122-125). ont acquis une grande renommée et qui ont été publiés à maintes reprises dans la littérature sur '"art africain. Ces masques Katundu sortent en premier lieu lors de fêtes et d'événements séculiers où règnent la détente et la réjouissance.

Les masques du Kasaï sont peu connus à cause de leur caractère secret et mystérieux. Ils ne sortent que pour remplir des fonctions très précises. Leur apparition a presque toujours lieu après l'aube ou juste avant le crépuscule. Les masques dansent ou sortent à l'occasion de la guérison de malades, du renouvellement d'un village, des danses accompagnant les semailes du millet, de la construction d'une nouvelle case cheffale, de l'investiture d'un nouveau chef, et enfin, à l'occasion de l'initiation des jeunes adolescents.

Les danseurs masqués sont généralement perçus comme des incarnations d'ancêtres, mvumbi. En tant que forces sumaturelles ils sont censés pouvoir influencer la vie humaine. Comme l'indique Cole (1985: 19-25), ce sont donc des matérialisations ou des personnifications d'esprits. A travers la mascarade, le monde mystérieux naturel et surnaturel est introduit dans la communauté humaine. Les hommes masqués se révèlent en quelque sorte comme des intermédiaires entre la nature et la culture, entre le village et la forêt, entre l'humain et le surnaturel, entre les hommes et les femmes, entre les initiés et les non initiés. Le même pouvoir médiateur caractérise le chef politique qui se disntigue par sa capacité de maintenir l'équilibre entre les forces opposantes et contradictoires. En somme, les dirigeants politiques doivent leur importance à des qualités religieuses, économiques, morales, sociales et politiques. Les masques, en tant que forces régulatoires, constituent ainsi un prolongement du pouvoir et de l'autorité du chef politique.

Janzen \& Kauenhoven-Janzen (1975: 46) attirent l'attention sur la profonde divergeance fonctionnelle entre les masques du Kasaï et ceux de la région de Katundu. La décentralisation et la fragmentarisation politique au $\mathrm{Ka}$ saï auraient comme conséquence que les masques y sont activement impliqués dans la vie politique afin d'assurer l'ordre et l'équilibre. Les masques, en tant que représentation d'ancêtres, possèdent le pouvoir requis pour intervenir dans les affaires humaines. 
PETRIDIS, C. Gipogo et Pumbu a mfumu: masques du pouvoir cheffal chez les Pende du Kasaï. Rev. do Museu de Arqueologia e Etnologia, S. Paulo, 2:75-89, 1992.

Deux personnages masqués sont directement liés au pouvoir et à l'autorité politique: Gipogo et Pumbu a mfumu. Le masque Gipogo fait partie des mbuya jia kifutshi, masques du village, qui s'opposent aux mbuya jia mukanda, masques de circoncision, et aux mbuya jia ufumu, masques du pouvoir politique. Néanmoins, cette dernière catégorie aurait trait aux sculptures décorant la case cheffale. De ce fait, il n'est pas clair quelle est la place du Pumbu a mfumu, puisque ce type de masque est explicitement décrit comme un "masque du pouvoir cheffal" dans le catalogue Sura Dji (1982: 66).

Les deux masques ont leurs équivalents en région Katundu dans les masques Fumu et Pumbu. Ces types Katundu, représentant respectivement le chef et le guerrier, ne peuvent être distingués du point de vue morphologique. En effet, chez les Katundu on ne peut identifier la personnage incarné que par la représentation théatrâle. Par contre, les masques Gipogo et Pumbu a mfumu du Kasaï constituent deux styles distincts.

Le Gipogo est sans doute le type de masque du Kasaï le plus connu et le mieux représenté dans les collections occidentales. Il s'agit d'un masque cloche muni d'un prolongement mentonnier horizontal en forme de plateau discoïde. L'on pourrait établir une parenté formelle lointaine avec les masques en forme de heaume des Pende du Kwilu, des Kwese, des Mbala, des Suku et des Yaka. ${ }^{3}$ Selon Bourgeois (1990: 124), les masques cloches des Pende du Kasaï sont transitoires aux formes stylistiques répandues plus à l'est. $\mathrm{Si}$ entre le Kwango et le Kasaï la zone faciale est plutôt modeste en comparaison avec la masse crâniale, c'est la face qui domine chez les masques heaumes des peuplades à l'est. De Sousberghe (1954a: 80) écrit que le style

(3) Selon Bourgeois (1990: 124), un certain nombre de masques de la région entre le $\mathrm{Kwango}$ et le Kasaï ont des traits morphologiques qui dépassent les limites des styles locaux et "tribaux : Néanmoins, l'auter montre deux masques cloches des Pende du Kasaï qui ne ressemblent que très sommairement aux sculptures typiques de ce genre. Ces deux pièces font partie d'une série de masques qui fût récoltée en 1905 par un certain Frederick Starr et qui se trouve aujourd hui à l'American Museum of Natural History à New York (1990: ills. 28 et 29). du Gipogo est dû à "un substrat ethnique antérieur au Pende, au Kasaï (Bakete ?)".

D'autres traits sont par contre clairement d'inspiration tshokwe. Bastin (1961: 38) suppose que le masque cloche Gipogo a été créé postérieurement au masque pende homonyme triangulaire avec des superstructures en forme de couteaux. L'auteur reconnaît une influence stylistique tshokwe aussi bien dans des éléments constructifs que dans des éléments décoratifs. Ce sont surtout le plateau mentonnier et la décoration en forme de dents de scie qui rappellent certains types de masques tshokwe. Il est également important de noter que le nom Gipogo est parfois remplacé par Mukishi wa mutue. Or, chez les Tshokwe, la dénomination mukishi réfère à un esprit ancestral ou naturel incarné par un masque.

Il n'y a pourtant pas que la forme et la dénomination du masque qui renvoient aux Tshokwe, il en est de même pour sa fonction et sa signification. Le masque tshokwe Tshikungu ou Mukishi wa mwanangana représente le chef et symbolise le pouvoir ancestral, tout comme le Gipogo des Pende. De plus, la sculpture est gardée soigneusement dans une petite hutte et ne sort que dans des circonstances exceptionnelles afin d'assurer le bienêtre de la communauté entière. De ce fait, le Tshikungu est simultanément un mukishi, un esprit naturel ou ancestral incarné par un masque, et un hamba, un esprit naturel ou ancestral qui est le sujet d'un culte (Bastin, 1984: 40-42). ${ }^{4}$

La sculpture pende est conservée dans la case cheffale et fait partie du trésor, kifumu. Tout comme les autres objets rituels constituant ce trésor, le Gipogo aurait une influence directe sur le bien-être et la santé de la population. En outre, l'absence du masque causerait des maladies et des malheurs. D'autre part, plusieurs masques des Pende du Kasaï, dont le Gipogo, sont reproduits au sommet de pieux qui entourent la case cheffale.

Le danseur masqué représente le chef po-

(4) Les masques Gipogo et Tshikungu, en tant que sculptures sacrées par excellence, peuvent être comparés au Ngongo munene des Dinga (Kongo) et au Katatola des Lwena. Ces quatre types de masques sont des symboles de l'autorité déléguée par les ancêtres et leur exhibition est bienfaisante pour la vie de la communauté (Bastin, 1961: 38). 
litique et évoque le pouvoir ancestral. La sculpture en soi constitue un des insignes les plus importants du chef et est considérée comme une source de fécondité pareille au chef même. Le caractère ambigu et ambivalent de l'homme politique, en tant que donneur de vie et de mort, est suggéré par les couteaux ou les épées, pogo ya mwil ou pogo ya kusa, que le danseur tient dans les mains. Ces couteaux peuvent sans doute être interprétés comme les prolongements de la force physique du chef. De nos jours, ils sont souvent remplacés par des fusils d'origine européenne. Parfois, le danseur porte des chasse-mouches comme insignes de prestige.

Les mêmes idées d'agressivité et de force sont exprimées par la couleur rouge du masque, couleur derivée du tukula, poudre rouge du Pterocarpus, ainsi que par les peaux d'animaux attachées à la ceinture du danseur. II s'agit très probablement de peaux de la genette (Genetta genetta ou G. tigrina ?), shimba. La genette est considérére comme l'animal cheffal par excellence: normalement il n'y a que les chefs qui peuvent porter ou posséder des peaux de shimba. D'ailleurs, des peaux de différents animaux font également partie du trésor, kifumu. De Sousberghe (1959: 65) mentionne que le masque est orné d'une peau de singe et d'une coiffe de plumes. S'agit-il de plumes rouges du nduwa, Lady Ross' touraco (Mussophaga rossae), ou bien de plumes bleues du touraco géant ou boulicoco, kolomvu (Corythaeola cristata) ? Les plumes du nduwa symbolisent le succès dans les épreuves et le meurtre d'un animal ou d'un ennemi, tandis que le kolomvu, oiseau qui aime les retraites obscures dans la forêt, symbolise la nuit et la mort.

Le personnage sort surtout dans le cadre de la guérison de malades. De Sousberghe (1959: 65) a été plus précisément témoin de la guérison d'une femme avec un enfant malade dans le village Ngimbu près de Njinji, lorsqu'un petit masque triangulaire nommé Pota accompagnait le masque cloche. Le Gipogo apparaît également lors des danses en honneur du renouvellement d'un village. Malutshi Mudiji-Selnge (1981: 229-230) parle de cultes ancestraux et de cérémonies de circoncision.

Le masque Pumbu a mfumu constitue un style particulier. Il s'agit d'un grand mas- que demi-heaume avec des yeux tubulaires et un prolongement mentonnier vertical de forme rectangulaire. Ce prolongement est décoré sur toute sa surface de motifs géométriques. Nous ne connaissons que quelques exemplaires de ce type de masque et apparemment peu de masques ont quitté le territoire zaïrois. Le musée de Tervuren n'en possède par exemple qu'un spécimen. Cet exemplaire ressemble beaucoup à un masque du musée de Berg en Dal aux Pays-Bas. Il est plausible que ces deux sculptures soient l'oeuvre d'un même sculpteur. L'hypothèse d'une même main ou d'un même atelier peut aussi être appliquée à un certain nombre de masques dispersés dans le monde entier (Institut des Musées Nationaux du Zaïre, Kinshasa; National Museum of African Art, Washington; collection P. \& R. Tishman).

Le nom Pumbu a mfumu est mentionné par De Sousberghe (1959: 61) en rapport avec un masque récolté par Robert Verly pour la 'Biennale des Arts Congolais' à Luluabourg en 1957, ainsi qu'avec une pièce photographiée in situ par Michel Huet avant 1958. Selon le dire de Verly, la sculpture a souvent, "au lieu de plumes, au sommet de la tête des flèches qui se croisent horizontalement".

Généralement, le personnage masqué Pumbu a mfumu est considéré comme l'incarnation de l'ancêtre cheffal et parfois comme la représentation du bourreau ou de l'exécuteur. Il est intéressant de noter que De Sousberghe (1959: 31), en traitant les masques des Katundu, écrit que le type Pumbu de ces derniers pourrait être considéré comme la transposition d'un masque de la classe des minganji. Jadis, ces masques minganji en fibres participaient à l'exécution d'un ennemi ou d'un passant à l'occasion de l'investiture d'un nouveau chef.

L'auteur des légendes du catalogue Sura Dji (1982: 66-Lema Gwete ?) note que le masque Pumbu a mfumu n'est porté que par le lemba ou chef de clan lors des funérailles d'un chef ou d'un notable ou dans le cas de certaines maladies. Outre le lien avec les mahamba, tiges et arbres que l'on plante autor de la case cheffale en honneur des ancêtres, le personnage jouerait également un rôle dans le fondement d'un nouveau village.

D'autre part, De Sousberghe (1959: 6162) raconte qu'en 1952, il a eu la chance de 
PETRIDIS, C. Gipogo et Pumbu a mfumu: masques du pouvoir cheffal chez les Pende du Kasaî. Rev. do Museu de Arqueologia e Etnologia, S. Paulo, 2:75-89, 1992.

voir deux masques géants au village Tshikunga-Katembo (Kasai). Ce ne fût qu'à condition qu'aucune photo n'en soit prise que le chef local consentit à les montrer. Les sculptures étaint nommées mbuya ya ufumu, signifiant "masques du pouvoir cheffal". L'un des masques portait le nom Pumbu et ressemblait quelque peu au Kakuungu des Yaka/Suku, tandis que l'autre spécimen était nommé Mbologoto et rappelait le Dakakulo des Kete voisins. Ces deux masques sortaient uniquement pour planter autour de la case cheffale les mahamba cités plus haut. Curieusement, les deux types de masques ne sont mentionnés dans aucun autre texte:"

Il existe également une variante sur le style Pumbu a mfumu habituel. Dans ce cas, le visage est presque triangulaire et la décoration plus sobre. Au lieu des yeux percés, on retrouve trous de chaque côté du nez. Un exemplaire de cette variante stylistique se trouve à l'Institut des Musées Nationaux du Zaïre à Kinshasa, un autre dans la collection de Harrison Eiteljorg à Indianapolis. Selon l'auter du cataloque Sura Dji (1982: 68), ce type de Pumbu a mfumu parait en cas de calamités, ainsi qu’à la fin des épreuves de la circoncision, lorsque chaque initié doit avaler une bouchée de manioc qui est placée sur la bouche du masque. Il est plutôt étrange que Malutshi Mudiji-Selnge (1981: 229-230) attribue le même rôle au masque cloche Gipogo.
Bien que nous ne disposions que de quelques renseignements concernant les deux masques, il est clair que le Gipogo et le Pumbu a mfumu incarnent le même idéal masculin politique. Les deux personnages glorifient et célèbrent le pouvoir politique afin de le protéger et de le sauvegarder. L'agressivité, la force et la violence, qui se reflètent dans la polychromie, dans l'iconographie et dans les attributs, sont des moyens efficaces dans l'exercice du pouvoir. La situation politique est représentée et actualisée par le biais de la mascarade et les masques renforcent la foi et la soumíssion de la population. En même temps, la mascarade contribue à la satisfaction des besoins esthétiques et émotionnels du public.

\section{Notes}

Nous tenons à remercier le Professeur $\mathrm{H}$. Burssens et Mme. E. Bruyninx pour l'aide qu'ils nous ont apportée tout au long de la préparation de notre mémoire de licence (Université de Gand, Belgique). Nous exprimons également notre reconnaissance à Mme. $\mathrm{H}$. van Geluwe qui nous a permis d'étudier de près la riche collection du Musée Royal de l'Afrique Centrale de Tervuren. Enfin, nous remercions les personnes et les institutions qui nous ont remis les photographies illustrant cet article. 


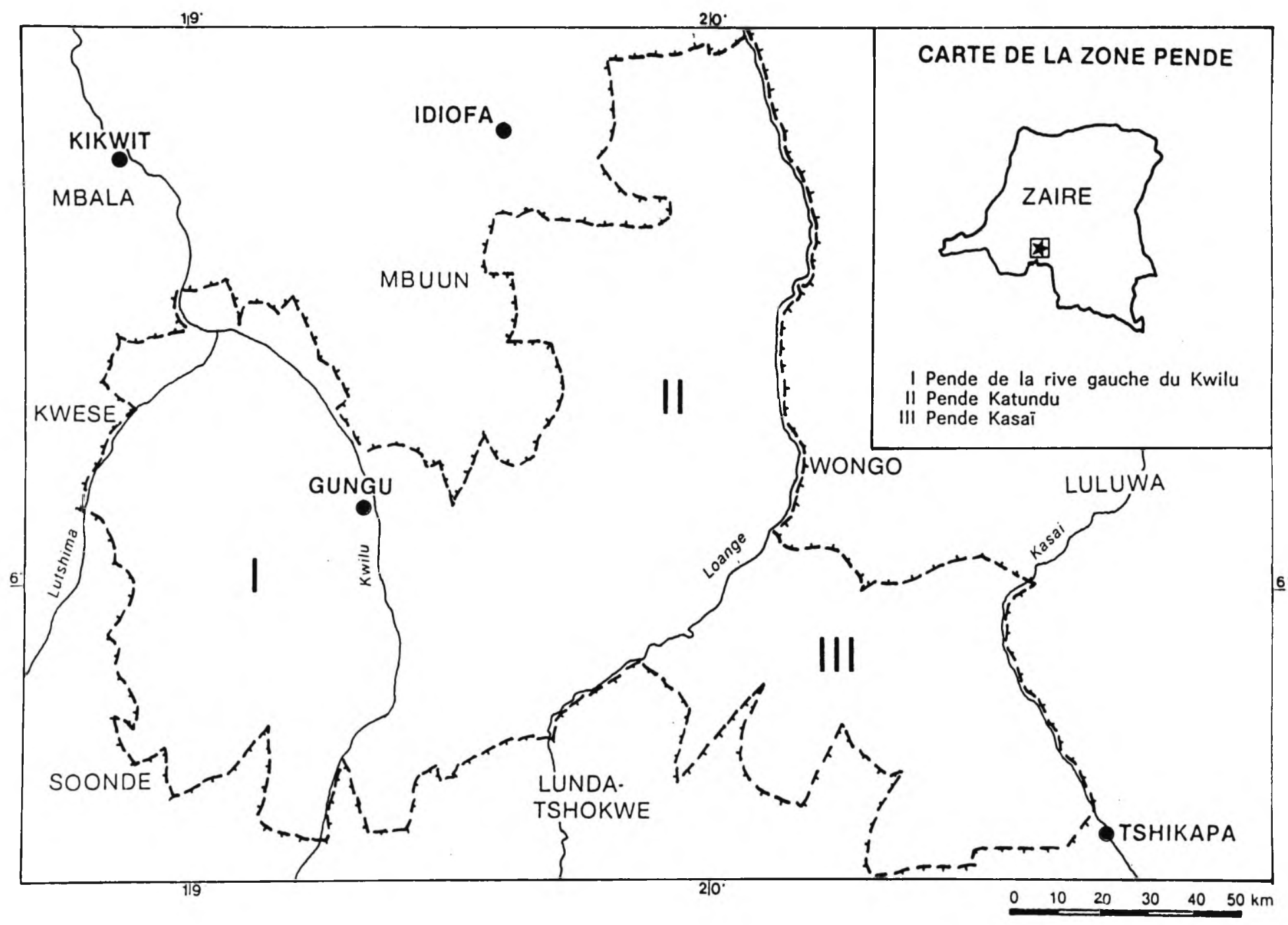


PETRIDIS, C. Gipogo et Pumbu a mfumu: masques du pouvoir cheffal chez les Pende du Kasaĩ. Rev. do Museu de Arqueologia e Etnologia, S. Paulo, 2:75-89, 1992.

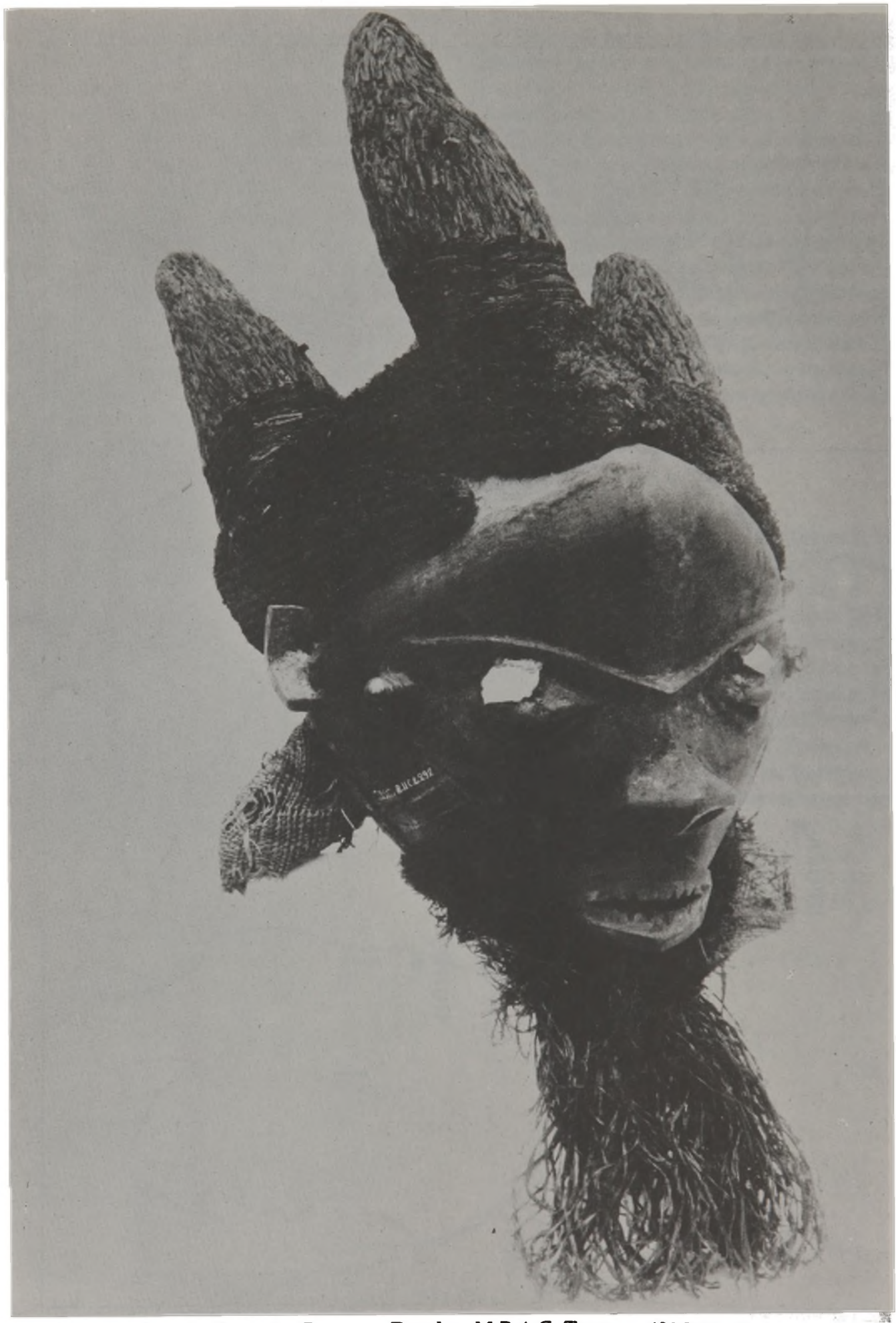

1 - Masque de style Katundu, Fumu ou Pumbu. M.R.A.C. Tervuren (32517). H. $23 \mathrm{~cm}$. Récolté par R. Wenner au Kwango (Bandundu, Kilembe, chefferie Mbushi) avant 1932. Photo: M.R.A.C. 
PETRIDIS, C. Gipogo et Pumbu a mfumu: masques du pouvoir cheffal chez les Pende du Kasaï. Rev. do Museu de Arqueologia e Emologia, S. Paulo, 2:75-89, 1992.

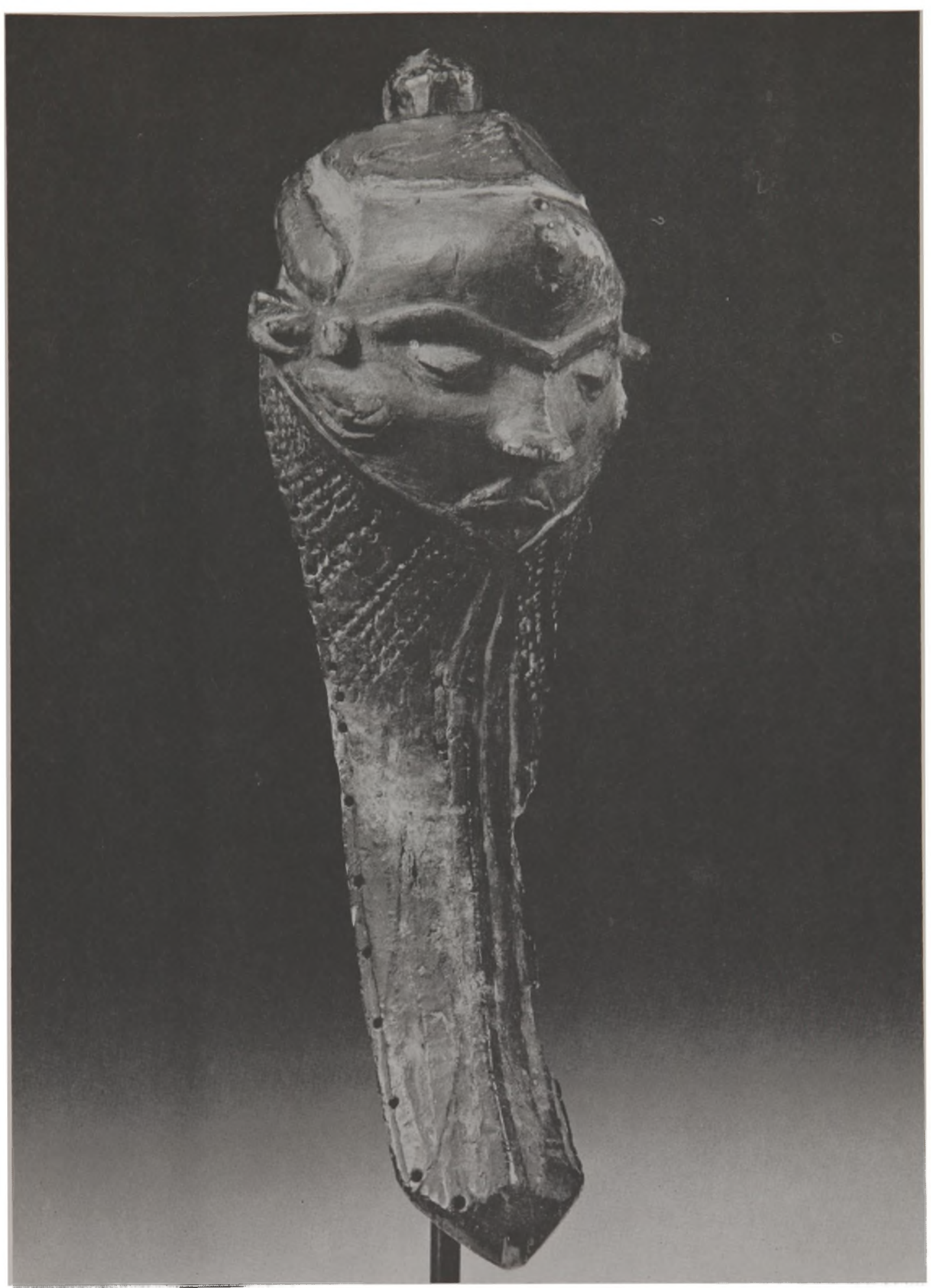

2 - Masque de style Katundu, Kiwoyo ou Muyombo. Collection privée. Dïsseldorf (Allemagne). H. 62 cm. Photo: E. Hesmerg. 
PETRIDIS, C. Gipogo et Pumbu a mfumu: masques du pouvoir cheffal chez les Pende du Kasaï. Rev. do Museu de Arqueologia e Etnologia, S. Paulo, 2:75-89, 1992.

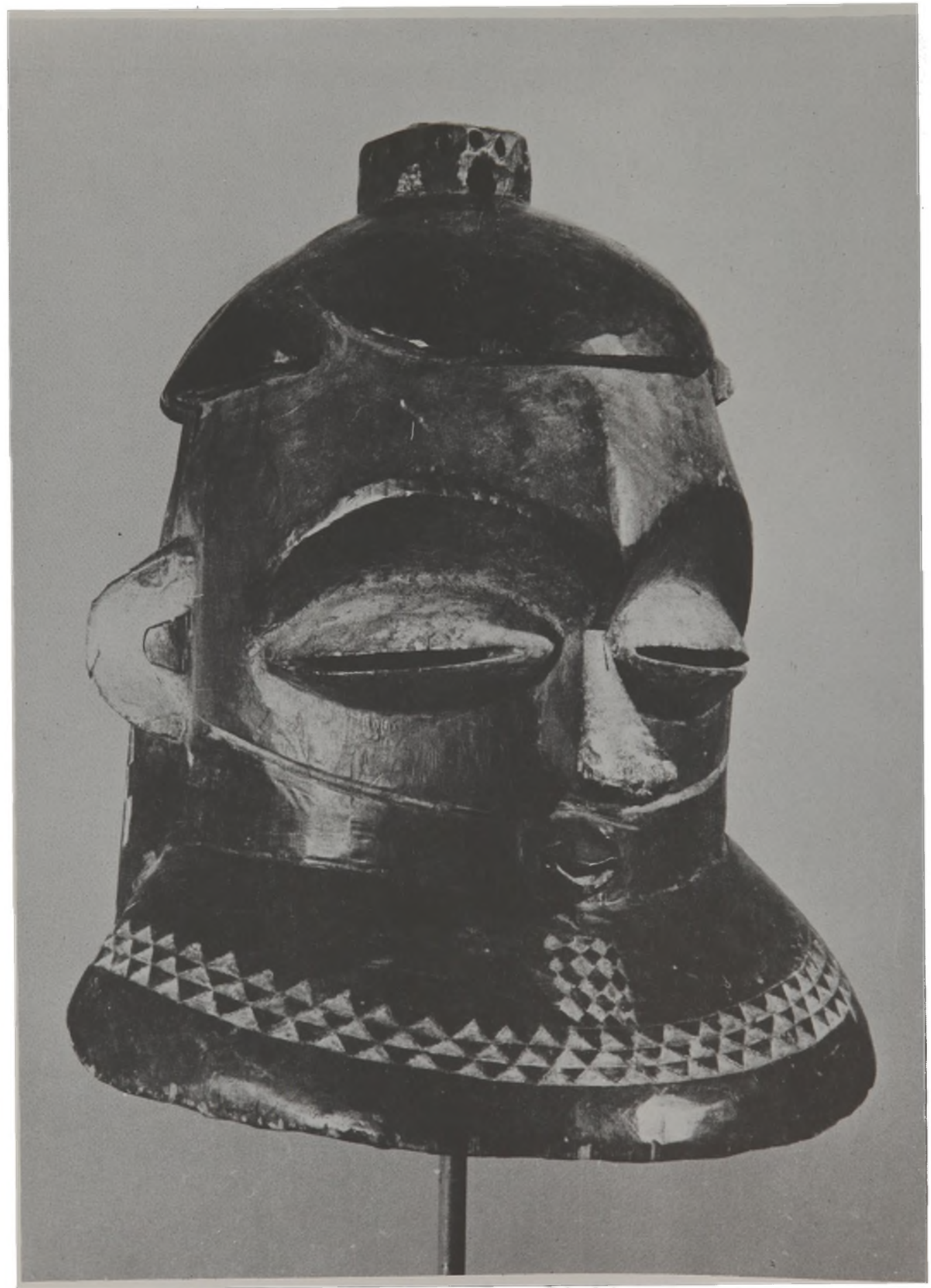

3 - Gipogo. M.R.A.C. Tervuren (43134). H. $27 \mathrm{~cm}$. Récolté par T. Fourche au Kasaï avant 1946. Photo: M.RA.C. 
PETRIDIS, C. Gipogo et Pumbu a mfumu: masques du pouvoir cheffal chez les Pende du Kasaï. Rev. do Museu de Arqueologia e Ernologia, S. Paulo, 2:75-89, 1992.

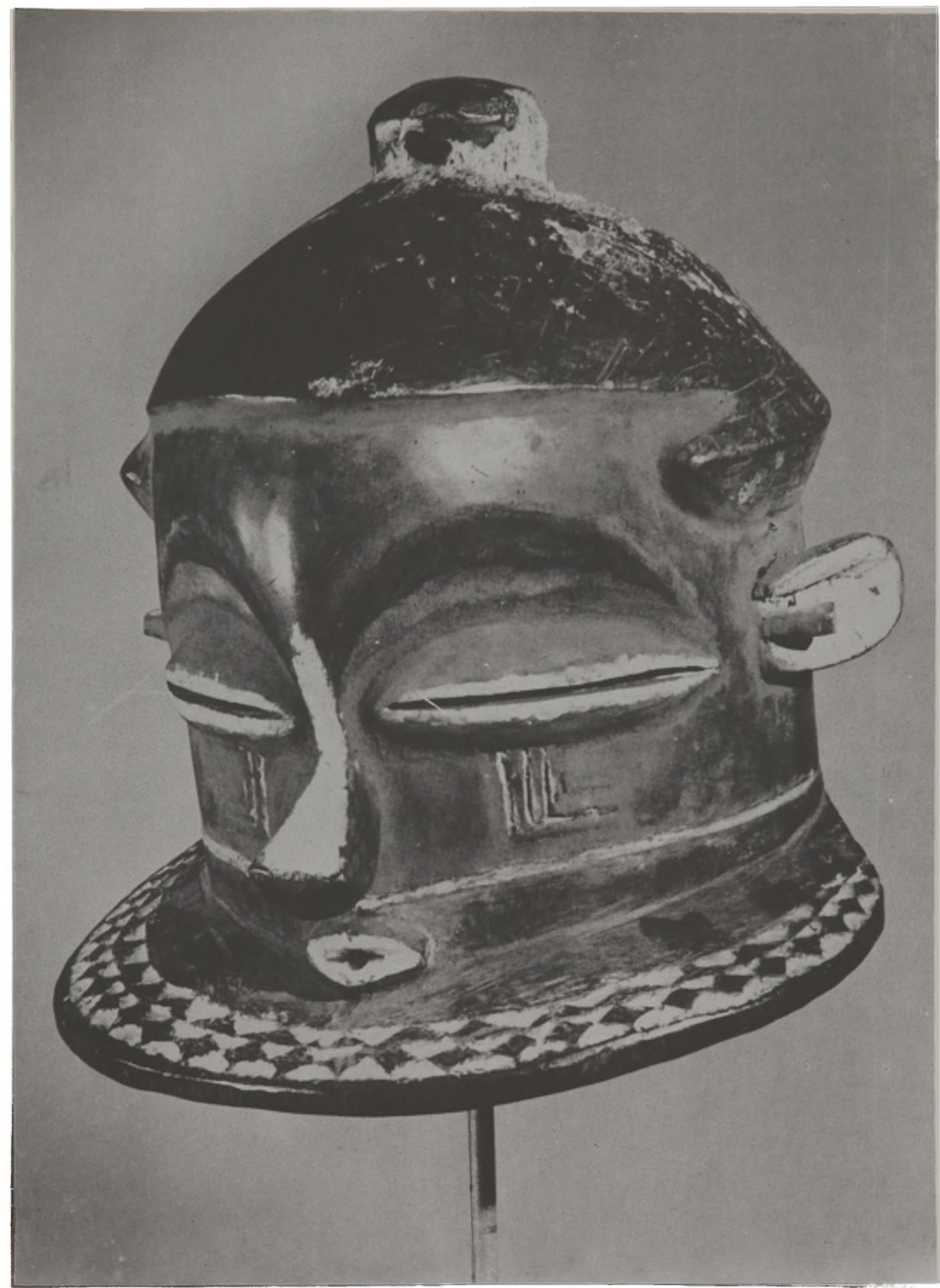

4 - Gipogo. M.R.A.C. Tervuren (53.74.5485). H. $28 \mathrm{~cm}$. Récolté par A. Maesen au Kasaï (zone Tshikapa, village Kindamba) en 1954. Sculpté par Ngolomi. Photo: M.R.A.C. 
PETRIDIS, C. Gipogo et Pumbu a mfumu: masques du pouvoir cheffal chez les Pende du Kasaï. Rev. do Museu de Arqueologia e Etnologia, S. Paulo, 2:75-89, 1992.

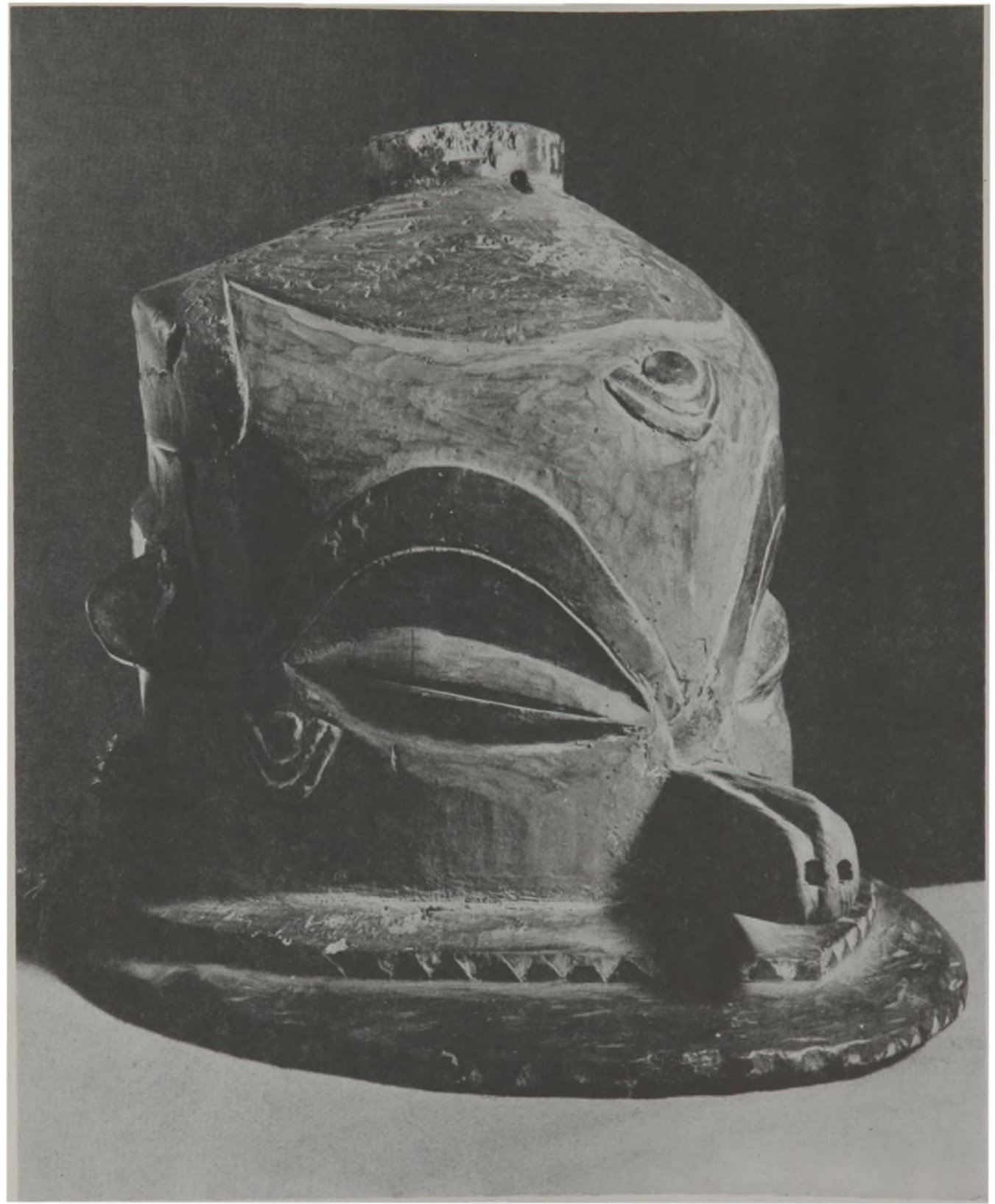

5 - Gipogo. Rautenstrauch-Joesi Museum, Kölñ (Alleminăgne) (48963). H. 27 cm. Ácquis par le musée en 1966. Ancienne collection Klaus Clausmeyer. Photo: Rautenstrauch-Joest Museum. 
PETRIDIS, C. Gipogo et Pumbu a mfumu: masques du pouvoir cheffal chez les Pende du Kasaï. Rev. do Museu de Arqueologia e Etnologia, S. Paulo, 2:75-89, 1992.

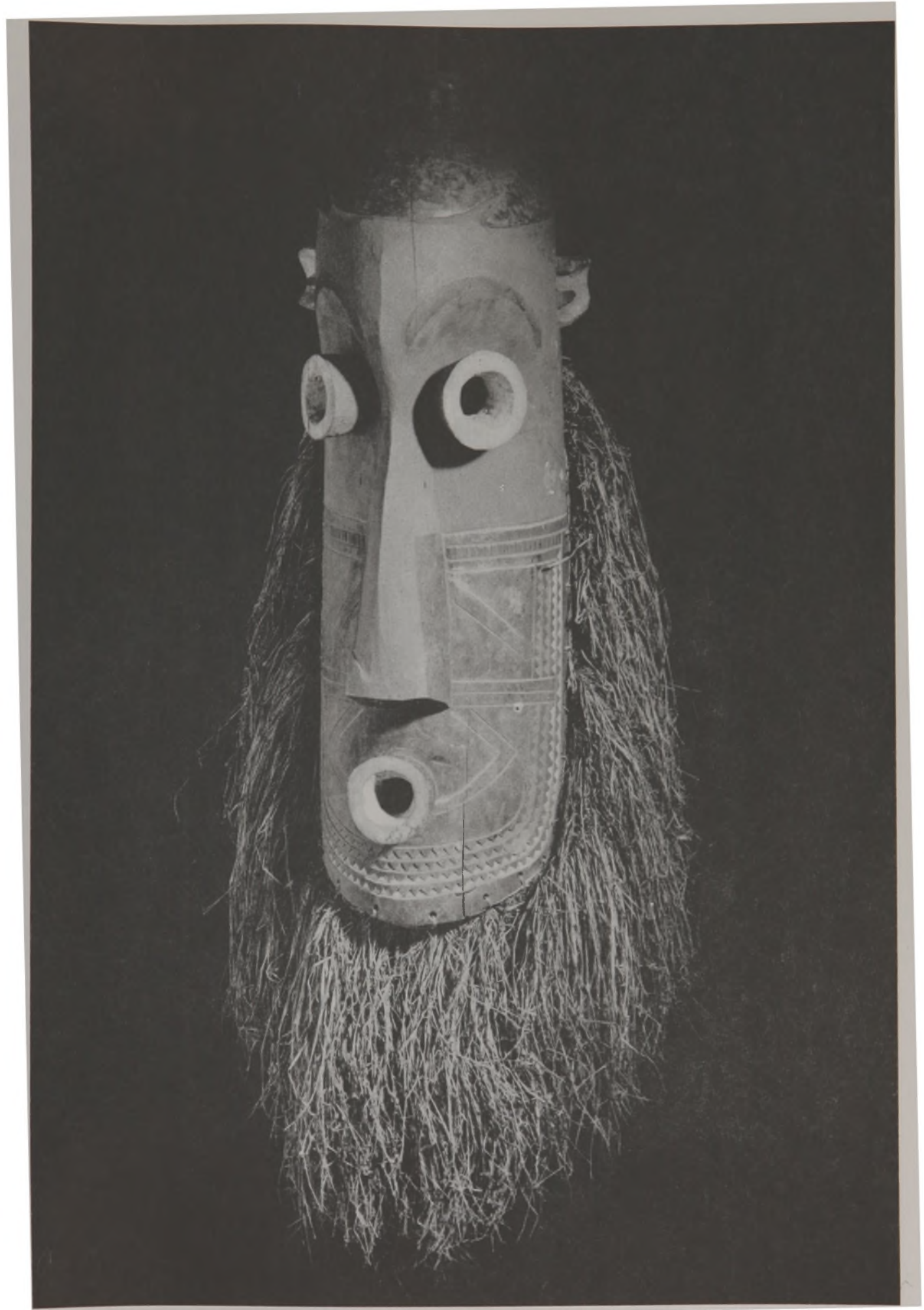

6- Pumbu a mfumu. Afrika Museum, Berg en Dal (Pays-Bas) (72.1). H. $77 \mathrm{~cm}$. Récolté par P. Timmermans au Kasaï (village Kayala Holo ?) avant 1966. Photo: Afrika Museum. 
PETRIDIS, C. Gipogo et Pumbu a mfumu: masques du pouvoir cheffal chez les Pende du Kasaï. Rev. do Museu de Arqueologia e Etnologia, S. Paulo, 2:75-89, 1992.

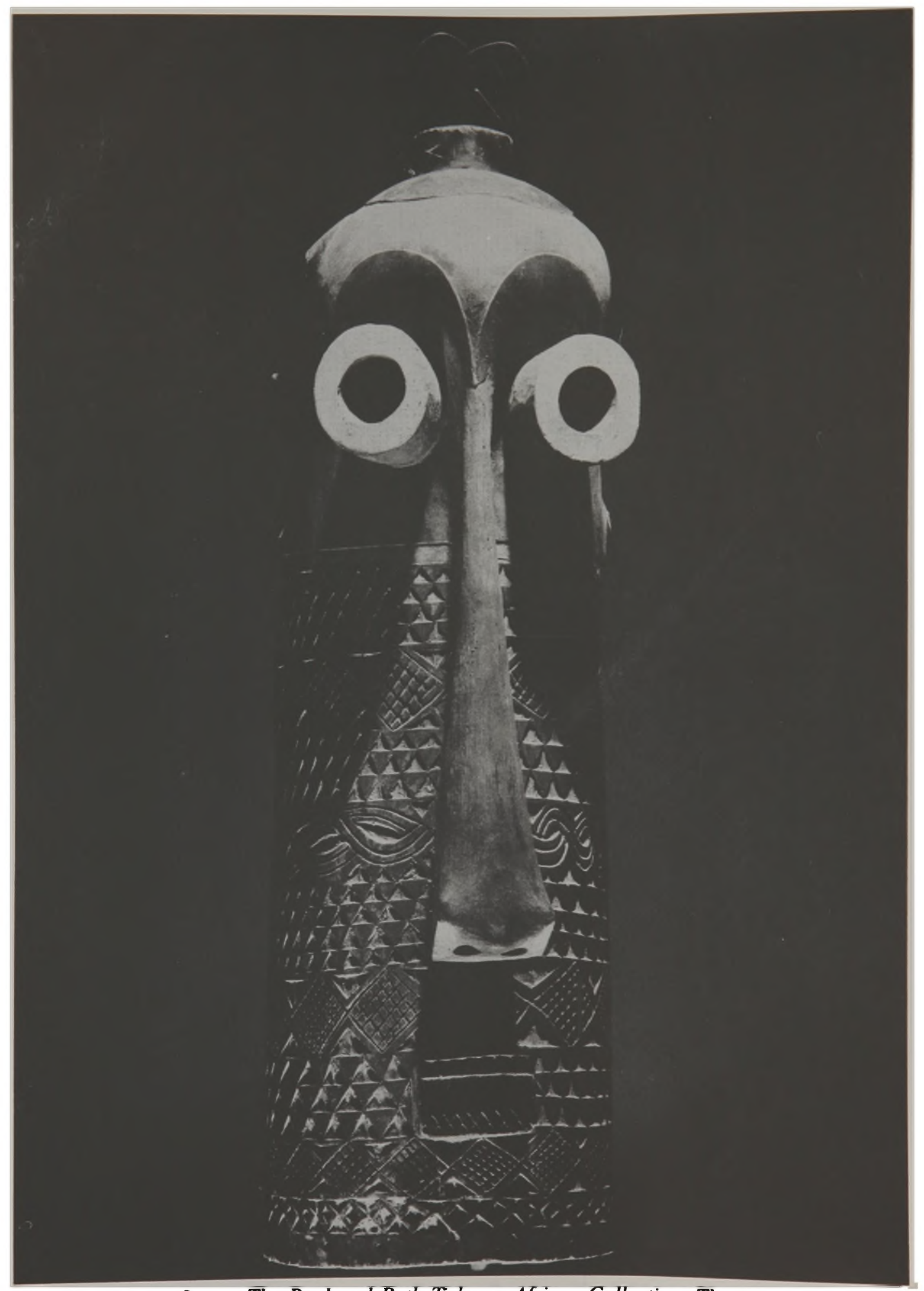

7 - Pumbu a mfumu. The Paul and Ruth Tishman African Collection, The Disney Co. $\mathrm{H}$. 74,9 cm. Photo: Geza Fekete. 
PETRIDIS, C. Gipogo et Pumbu a mfumu: masques du pouvoir cheffal chez les Pende du Kasaī. Rev. do Museu de Arqueologia e Etnologia, S. Paulo, 2:75-89, 1992.

PETRIDIS, C. Gipogo and Pumbu a mfumu: chiefly power masks among the Pende of Kasaï. Rev. do Museu de Arqueologia Etnologia, S. Paulo, 2:75-89, 1992.

ABSTRACT: The aim of this article is to indicate the political dimension of two wooden mask types of the Kasaï Pende. Attention is drawn to both formal and functional aspects. Research has been dictated by the available literature on the subject and by a morphological analysis of a vast number of masks. Although the chief's houses are the most impressive artistic achievements with regard to Pende chiefly power, forceful dominant behaviour is expressed in a dynamic way in Pende masquerades. Among the Kasai or eastern Pende the Gipogo and Pumbu a mfumu mask types glorify and celebrate political leadership in order to saveguard the existing power relations. As mediators between opposite realms and as regulating forces, the maskers appear as extensions of the chief's authority

UNITERMS: Zaïre - Kasaï Pende - masks - political power.

\section{References bibliographiques}

BASTIM, M-L. Un masque en cuivre martelé des Kongo du nord-est de l'Angola. Africa-Tervuren 7(2): 29-40, 1961.

- Ritual masks of the Chokwe. African Arts 17(4): 40-44; 92-93; 95, 1984.

BITTREMIEUX, L. De Baphende's van Luanda (Opper-Kasai). Congo $1(1): 27-39 ; 1$ (2): 153-181, 1939.

BOURGEOIS, A. P. Helmet-shaped masks from the Kwango-Kwilu region and beyond. In lowa studies in African art: the Stanley conferences at the University of Iowa. Volume III. Art and initiation in Zaïre. C. D. Roy (ed.). Iowa: The University of Iowa, 1990: 121-136.

COLE, H. M. Introduction: the mask, masking, and masquerade arts in Africa. In I am not myself The art of African masquerade. H. M. Cole (ed.). Los Angeles: Regents of the University of California, 1985: 15-27.

-. Icons. Ideals and power in the art of Africa. Washington: Smithsonian Institution Press, 1989.

DE SOUSBERGHE, L. Cases cheffales sculptées des ba-Pende. Bulletin de la Société Royale Belge d'Anthropologie et de Préhistoire 65: 75-81, 1954a.

—. Etuis péniens ou gaines de chasteté chez les baPende. Africa 24(3): 214-219, $1954 \mathrm{~b}$.

- L'art pende. Bruxelles: Académie Royale de Belgique, Classe des Beaux-Arts, IX, 2, IX, 1959.

_. Les Pende. Aspects des structures sociales et politiques." In Miscellanea ethnographica (L. de
Sousberghe, B. Crine-Mavar, A. Doutreloux, J. De Loose). Tervuren: Annales du Musée Royal d'Afrique Centrale, Sciences Humaines, 46: 1-78, 1963.

FRASER, D. \& H. M. COLE. Art and leadership: an overview. In African art and leadership. D. Fraser \& H. M. Cole (eds.). Madison: The University of Wisconsin Press, 1972: 295-328.

JANZEN, J. \& R. KAUENHOVEN-JANZEN. Pende masks in Kauffman museum. African Arts 7(4): 4447, 1975.

MALUTSHI MUDIJI-SELNGE. 141. Mask (Kiphoko). In For spirits and kings. African art from the Tishman Collection. S. Vogel (ed.). New York: The Metropolitan Museum of Art, 1981: 229-230.

McLEOD, M. Verbal elements in West African art. Quaderni Poro 1: 85-102, 1976.

PETRIDIS, C. De houten maskers van de Pende (Zaïre: Lutshima-Kasai). Gent: Universiteit Gent (mémoire de licence inédit/unpublished licentiate's thesis), 1990-91.

-. Wooden masks of the Kasai Pende. In Working Papers in Ethnic Art 6. H. Burssens, E. Bruyninx \& R. Haeseryn (eds.). Gent: Universiteit Gent (à paraître/forthcoming).

SURA DJI. Sura Dji. Visages et racines du Zaïre. Paris: Musée des Arts Décoratifs, 1982.

VANDENHOUTE, J. P. Beschouwingen over het thema kunst en maatschappij. Tijdschrift voor Sociale Wetenschappen 5(1): 3-26, 1960. 